\title{
SUPERPOSITION OF FUNCTIONS IN SOBOLEV SPACES OF FRACTIONAL ORDER. A SURVEY
}

\author{
WINFRIED SICKEL \\ Mathematische Fakultät, Friedrich-Schiller-Universität Jena \\ D-O-6900 Jena, Germany
}

0. Introduction. The present paper is concerned with the study of the nonlinear operator

$$
T_{G}: f \rightarrow G(f),
$$

where $G: \mathbb{R}^{1} \rightarrow \mathbb{R}^{1}$ is a given function and $f$ is taken from a generalized Sobolev space $H_{p}^{s}\left(\mathbb{R}^{n}\right)$ (cf. Section 1 for definitions). Operators of that type are called superposition or Nemytskilu operators, and play a crucial role in nonlinear analysis. Our aim here is to describe under what conditions one can establish an embedding of the form

$$
T_{G}\left(H_{p}^{s_{0}}\left(\mathbb{R}^{n}\right)\right) \hookrightarrow H_{p}^{s_{1}}\left(\mathbb{R}^{n}\right), \quad s_{1} \leq s_{0} .
$$

Since the paper of Dahlberg [7] it is known that one cannot expect $s_{0}=s_{1}$ in general. The loss of smoothness under the superposition, even in the case $G \in C^{\infty}\left(\mathbb{R}^{1}\right)$, depends on the dimension $n$ as well as on the smoothness and integrability properties of $f \in H_{p}^{s}\left(\mathbb{R}^{n}\right)$. This behaviour of $T_{G}$ will be explained in what follows. Let us mention that all results are presented in the framework of the scale $H_{p}^{s}\left(\mathbb{R}^{n}\right)$. However, they remain true if one replaces $H_{p}^{s}\left(\mathbb{R}^{n}\right)$ by Slobodetskiu spaces $W_{p}^{s}\left(\mathbb{R}^{n}\right)$, the more general Besov spaces $B_{p, q}^{s}\left(\mathbb{R}^{n}\right)$ or the Triebel-Lizorkin spaces $F_{p, q}^{s}\left(\mathbb{R}^{n}\right)$ (a generalization of $H_{p}^{s}\left(\mathbb{R}^{n}\right)$, cf. Triebel [30]). Let us refer also to the recent monograph by Appell and Zabreiko [2], where such problems are investigated from a somewhat different point of view.

This survey summarizes recent results obtained by the Jena research group on function spaces around $\mathrm{H}$. Triebel. It is based on a lecture given at the Stefan Banach International Center in Warsaw in November 1990.

1. Sobolev spaces of fractional order. The symbol $\mathbb{R}^{n}$ represents the Euclidean $n$-space, by $\mathbb{Z}$ we denote the set of all integers, and by $\mathbb{N}$ all natural 
numbers. Let $S\left(\mathbb{R}^{n}\right)$ be the Schwartz space of all complex-valued rapidly decreasing and infinitely differentiable functions on $\mathbb{R}^{n}, S^{\prime}\left(\mathbb{R}^{n}\right)$ the set of all tempered distributions on $\mathbb{R}^{n}, F$ and $F^{-1}$ the Fourier transform and its inverse on $S^{\prime}\left(\mathbb{R}^{n}\right)$, respectively.

Definition. Let $1<p<\infty$ and $s \geq 0$. The Sobolev space $H_{p}^{s}\left(\mathbb{R}^{n}\right)$ of fractional order $s$ is the set of all $f \in L_{p}\left(\mathbb{R}^{n}\right)$ such that

$$
\left\|f\left|H_{p}^{s}\left(\mathbb{R}^{n}\right)\|=\| F^{-1}\left(1+|\xi|^{2}\right)^{s / 2} F f\right| L_{p}\left(\mathbb{R}^{n}\right)\right\|<\infty .
$$

R e mark 1. We follow here the classical approach of Aronszajn-Smith [3] and Calderón [5]. Sometimes the spaces $H_{p}^{s}\left(\mathbb{R}^{n}\right)$ are also called Liouville spaces (in particular in the Russian literature) or Bessel-potential spaces.

Remark 2. A more explicit description of $H_{p}^{s}\left(\mathbb{R}^{n}\right)$ can be obtained with the help of differences. We put

$$
\left(\Delta_{h}^{1} f\right)(x)=f(x+h)-f(x), \quad\left(\Delta_{h}^{l} f\right)(x)=\Delta_{h}^{1}\left(\Delta_{h}^{l-1} f\right)(x), \quad l=2,3, \ldots
$$

Then we have with $l>s>0, l \in \mathbb{N}$,

$$
\begin{aligned}
f \in H_{p}^{s}\left(\mathbb{R}^{n}\right) \Leftrightarrow f \in L_{p}\left(\mathbb{R}^{n}\right) \text { and } \\
\left\|f\left|L_{p}\left(\mathbb{R}^{n}\right)\|+\|\left(\int_{0}^{1} r^{-2 s}\left(\int_{\{h:|h| \leq 1\}}\left|\Delta_{r h}^{l} f(\cdot)\right| d h\right)^{2} \frac{d r}{r}\right)^{1 / 2}\right| L_{p}\left(\mathbb{R}^{n}\right)\right\|<\infty .
\end{aligned}
$$

Moreover, the expression in (1.2) yields an equivalent norm in $H_{p}^{s}\left(\mathbb{R}^{n}\right)$ (cf. Triebel [30]).

Basic properties. This scale generalizes the classical Sobolev spaces in a natural way:

(i) $H_{p}^{s}\left(\mathbb{R}^{n}\right)$ equipped with the norm in (1.1) is a Banach space,

(ii) $H_{p}^{m}\left(\mathbb{R}^{n}\right)=W_{p}^{m}\left(\mathbb{R}^{n}\right), m=1,2, \ldots$,

(iii) $H_{p}^{s_{0}}\left(\mathbb{R}^{n}\right) \hookrightarrow H_{p}^{s_{1}}\left(\mathbb{R}^{n}\right) \hookrightarrow H_{p}^{0}\left(\mathbb{R}^{n}\right)=L_{p}\left(\mathbb{R}^{n}\right)$ if $s_{0} \geq s_{1} \geq 0$ ("ฯ" always means continuous embedding),

(iv) $f \in H_{p}^{s}\left(\mathbb{R}^{n}\right)$ implies $\partial f / \partial x_{i} \in H_{p}^{s-1}\left(\mathbb{R}^{n}\right), i=1, \ldots, n$, if $s \geq 1$,

(v) $H_{p}^{s}\left(\mathbb{R}^{n}\right) \hookrightarrow L_{\infty}\left(\mathbb{R}^{n}\right) \Leftrightarrow H_{p}^{s}\left(\mathbb{R}^{n}\right) \hookrightarrow C\left(\mathbb{R}^{n}\right) \Leftrightarrow s>n / p$ (cf. Fig 1 ).

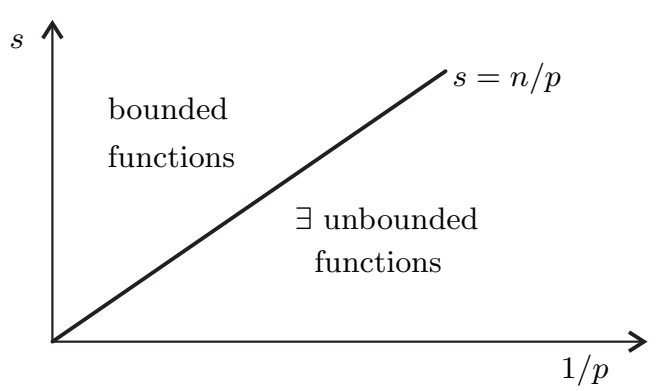

Fig. 1 
For (i)-(v) we refer to [30].

Finally, we consider two distinguished families of functions. Let $\Psi$ be a smooth cut-off function supported around zero and let $\alpha>0$. Then we define

$$
\begin{aligned}
& g_{\alpha}(x)=\Psi(x)|x|^{\alpha}, \\
& f_{\alpha}(x)=\Psi(x)|x|^{-\alpha} .
\end{aligned}
$$

It is known (cf. Stein [26], Triebel [30]) that

$$
\begin{aligned}
& g_{\alpha} \in H_{p}^{s}\left(\mathbb{R}^{n}\right) \Leftrightarrow s<n / p+\alpha, \\
& f_{\alpha} \in H_{p}^{s}\left(\mathbb{R}^{n}\right) \Leftrightarrow s<n / p-\alpha .
\end{aligned}
$$

In particular, the family $g_{\alpha}$ shows great similarity between measuring smoothness in the $H_{p}^{s}$-scale and in the $C^{s}$-scale (Hölder spaces).

If there is no danger of confusion we shall omit $\mathbb{R}^{n}$ in notations.

2. Boundedness of superposition operators. Our programme is to discuss the following three principal cases for the outer function $G$ :

(i) $G(t)=t^{m}, m=2,3, \ldots$,

(ii) $G(t)=|t|^{\mu}, \mu>1$,

(iii) $G(t) \in C^{\infty}\left(\mathbb{R}^{1}\right)$.

To do this we follow the way in which the pertinent results were proved. As we shall see the most striking feature will be the different behaviour of $T_{G}$ for bounded and unbounded functions. In this survey much attention is paid to describe the embedding (1.2) with proper inequalities.

2.1. Powers of $f$. First we investigate powers $f^{m}$ of $f$. It is a nonlinear problem, of course, but we can deal with it as a linear one, considering the family of operators

$$
T_{\left[g_{1}, \ldots, g_{m-1}\right]}(f)=\left(g_{1} \circ \ldots \circ g_{m-1}\right) \circ f, \quad f \in H_{p}^{s},
$$

where $g_{1}, \ldots, g_{m-1} \in H_{p}^{s}$ are fixed functions. Nowadays this problem is well understood. It is the problem of pointwise multipliers with respect to $H_{p}^{s}$.

TheOREM 1 ([23]). Let $m=2,3, \ldots$

(i) Let $s>n / p$. Then there exists a constant $c$ such that

$$
\left\|f^{m}\left|H_{p}^{s}\|\leq c\| f\right| H_{p}^{s}\right\|^{m} \quad \text { for all } f \in H_{p}^{s} .
$$

(ii) Let $0<s<n / p$. Let

$$
s_{m}=s-(m-1)(n / p-s)>0 .
$$

Then there exists a constant $c$ such that

$$
\left\|f^{m}\left|H_{p}^{s_{m}}\|\leq c\| f\right| H_{p}^{s}\right\|^{m} \quad \text { for all } f \in H_{p}^{s} .
$$

Remark 3. Whereas for bounded functions $(s>n / p)$ the result shows a good correspondence to that in the case of Hölder spaces $C^{s}$, the second part of Theorem 1 requires some further comments. Since $\left(f_{\alpha}\right)^{m}$ is of the same type as 
$f_{\alpha}$, but with a local singularity of order $m \alpha$, we can apply (1.6) to both functions. This yields $f_{\alpha}^{m} \in H_{p}^{r} \Leftrightarrow r<n / p-\alpha m$. For $f_{\alpha} \in H_{p}^{s}, \alpha \uparrow(n / p-s)$ we get

$$
r \leq n / p-m(n / p-s)=s-(m-1)(n / p-s)=s_{m} .
$$

This shows that each multiplication leads to a loss of smoothness of order $n / p-s$. Also the condition (2.2) can be interpreted with the help of the family $f_{\alpha}$. The inequality $s_{m}>0$ simply ensures $f_{\alpha}^{m} \in L_{p}=H_{p}^{0}$.

Remark 4. The statement (i) is a simple consequence of the fact that $H_{p}^{s}$, $s>n / p$, forms a multiplication algebra, a famous result of Strichartz [27]. The second statement in Theorem 1 was proved by Yamazaki [32] with the help of the paramultiplication principle. For a more detailed description (also in case $s=n / p)$ and further references we refer to the survey [23].

2.2. The real powers $|f|^{\mu}, \mu>1$. A new phenomenon appears when investigating $G(t)=|t|^{\mu}, \mu>1$, as the outer function. The finite smoothness of $|t|^{\mu}$ leads to a restriction on the smoothness of the superposition $G(f)$.

TheOREM 2 ([20], [24]). Let $\mu>1$.

(i) Let $n / p<s<\mu$. Then there exists a constant $c$ such that

$$
\left\|| f | ^ { \mu } | H _ { p } ^ { s } | \left|\leq c\left\|f \mid H_{p}^{s}\right\|^{\mu} \quad \text { for all } f \in H_{p}^{s} .\right.\right.
$$

(ii) Let $0<s<n / p$. Let

$$
0<s_{\mu}=s-(\mu-1)(n / p-s)<\mu .
$$

Then there exists a constant $c$ such that

$$
\left\||f|^{\mu}\left|H_{p}^{\mu}\|\leq c\| f\right| H_{p}^{s}\right\|^{\mu} \quad \text { for all } f \in H_{p}^{s} .
$$

Remark 5. For (ii) we can argue as in Theorem 1: again using the family $f_{\alpha}$ one derives that (2.5) ensures that $T_{\mu}^{*}: f \rightarrow|f|^{\mu}$ maps $H_{p}^{s}$ into $L_{p}$.

Remark 6. Part (i) is a consequence of a more general result proved by Runst [20]. A proof of (ii) may we found in Sickel [24]. Partial results may also be found in Triebel [31] and Edmunds-Triebel [9].

$A$ remark on the proof and a first generalization. In both cases the proof is based on the use of the Taylor expansion of $G(t)=|t|^{\mu}, \mu>1$. The estimate of the Taylor polynomial reduces to an application of Theorem 1. To obtain an estimate of the remainder one has to investigate the integral means

$$
\left(I_{k}^{\mu} f\right)(x)=\int_{|z| \leq 2^{-k}}|f(x+z)-f(x)|^{\mu} d z, \quad k \in \mathbb{Z} .
$$

In Runst [20] and Sickel [24] different estimates for these means were derived by using maximal-function techniques (Fefferman-Stein-Peetre maximal inequality, Hardy-Littlewood maximal inequality). 
However, only the following qualitative properties of $G(t)=|t|^{\mu}$ are used:

$$
\begin{gathered}
G: \mathbb{R}^{1} \rightarrow \mathbb{R}^{1}, \\
\left|G^{(l)}(t)\right| \leq c_{l}|t|^{\mu-l}, \quad l=0, \ldots, N, N \in \mathbb{N}, \\
\sup _{t_{0} \neq t_{1}} \frac{\left|G^{(N)}\left(t_{1}\right)-G^{(N)}\left(t_{0}\right)\right|}{\left|t_{1}-t_{0}\right|^{\tau}} \leq c<\infty, \quad \tau+N=\mu, 0<\tau \leq 1 .
\end{gathered}
$$

A simple reformulation of the conditions (2.8)-(2.10) is given by

$$
G \text { is } N \text { times continuously differentiable, }
$$

$$
\begin{gathered}
G^{(l)}(0)=0, \quad l=0, \ldots, N, \\
G^{(N)} \in \operatorname{Lip} \tau,
\end{gathered}
$$

where the Lipschitz space $\operatorname{Lip} \tau$ is characterized by (2.10). To make a composition $G(f)$ meaningful, we restrict ourselves to real-valued functions $f$.

Definition. Let $1<p<\infty$ and $s \geq 0$. By $\widetilde{H}_{p}^{s}$ we denote the subspace of $H_{p}^{s}$ consisting of all real-valued functions $f \in H_{p}^{s}$, equipped with the norm (1.1).

TheOREM 3 ([20], [24], [25]). Let $G$ be a function such that (2.11)-(2.13) are satisfied for some $\mu>1$. Then Theorem 2 remains true if we replace $|f|^{\mu}$ by $G(f)$ and $H_{p}^{s}$ by $\widetilde{H}_{p}^{s}$.

2.3. The case $G \in C^{\infty}\left(\mathbb{R}^{1}\right), G(0)=0$. As usual, $C^{m}\left(\mathbb{R}^{1}\right)$ denotes the set of functions $f$ such that

(i) $f, \ldots, f^{(m)}$ are uniformly continuous,

(ii) $\left\|f\left|C^{m}\left(\mathbb{R}^{1}\right) \|=\max _{0 \leq l \leq m} \sup _{t \in \mathbb{R}^{1}}\right| f^{(l)}(t) \mid<\infty\right.$.

We put

$$
C^{\infty}\left(\mathbb{R}^{1}\right)=\bigcap_{m=1}^{\infty} C^{m}\left(\mathbb{R}^{1}\right)
$$

To overcome the restriction (2.12) in Theorem 3 one uses the splitting

$$
G(t)=\left(G(t)-\sum_{j=0}^{N} \frac{G^{(j)}(0)}{j !} t^{j}\right)+\sum_{j=0}^{N} \frac{G^{(j)}(0)}{j !} t^{j}=H_{N}(t)+P_{N}(t) .
$$

Then $P_{N}(f)$ is estimated by Theorem 1 , and $H_{N}(f)$ by Theorem 3 . If $G \in$ $C^{\infty}\left(\mathbb{R}^{1}\right), G(0)=0$, then $N$ and $\tau$ are at our disposal. If $n / p<s$ we choose $\mu>\max (1, s), \mu \downarrow \max (1, s)$. If $s<n / p$ the situation is more complicated. Both $\mu$ and $s_{\mu}$ are upper bounds for the smoothness of $G(f)$. Since $s_{\mu}$ decreases if $\mu$ increases the optimal choice is $s_{\mu}=\mu$. We have

$$
\mu=s_{\mu}=\frac{n}{p}-\mu\left(\frac{n}{p}-s\right) \Leftrightarrow \mu\left(\frac{n}{p}-s+1\right)=\frac{n}{p} \Leftrightarrow \mu=\frac{n / p}{n / p-s+1} .
$$

From this point of view the following result is not surprising. 
Theorem 4. Let $G \in C^{\infty}\left(\mathbb{R}^{1}\right), G(0)=0$.

(i) Let $s>n / p$. Then there exists a constant $c$ such that

$$
\left\|G(f) \mid H_{p}^{s}\right\| \leq c\left(\left\|f\left|H_{p}^{s}\|+\| f\right| H_{p}^{s}\right\|^{\max (1, s)}\right) \quad \text { for all } f \in \widetilde{H}_{p}^{s} .
$$

(ii) Let $1<s<n / p$. Let

$$
\varrho(s, n / p)=\varrho=\frac{n / p}{n / p-s+1} .
$$

Then there exists a constant $c$ such that

$$
\left\|G(f) \mid H_{p}^{\varrho}\right\| \leq c\left(\left\|f\left|H_{p}^{s}\|+\| f\right| H_{p}^{s}\right\|^{\varrho}\right) \quad \text { for all } f \in \widetilde{H}_{p}^{s} .
$$

(iii) Let $0 \leq s \leq 1$. Then there exists a constant $c$ such that

$$
\left\|G(f)\left|H_{p}^{s}\|\leq c\| f\right| H_{p}^{s}\right\| \quad \text { for all } f \in \widetilde{H}_{p}^{s} .
$$

Some comments. (i) The case $s>n / p$. With regard to this case there are numerous references. The first is Mizohata [15], who had discovered $T_{G}\left(\widetilde{H}_{2}^{s}\right) \hookrightarrow$ $H_{2}^{s}, s>n / 2$, in 1965. Fifteen years later Meyer [14] established $T_{G}\left(\widetilde{H}_{p}^{s}\right) \hookrightarrow H_{p}^{s}$ by using the elegant method of paradifferential operators. Inspired by Meyer's work there exist further extensions to the classes $B_{p, q}^{s}$ and $F_{p, q}^{s}$ (Runst [19]), to anisotropic spaces (Yamazaki [32]), and to weighted spaces (Marschall [13]). Runst [20] applied maximal function techniques to this problem. However, the simple structure of (2.14), including the exponents, seems to be new. Note that at least for the Sobolev spaces $H_{p}^{m}\left(=W_{p}^{m}\right)$ these exponents are optimal. We refer to Sickel [25].

(ii) The case $0 \leq s \leq 1$. Because our function $G$ is smooth one can apply the chain rule. Now, (2.17) is a simple consequence for $s=1$. If $0<s<1$ then (2.17) follows from (1.2). In case $s=0$ inequality (2.17) is again obvious.

(iii) The case $1<s<n / p$. First, note that the restriction on $s$ implies $1<\varrho<s$, so we have some loss of smoothness. The reason becomes clear by the following example. Again we use the family $f_{\alpha}$ defined in (1.4). We have

$$
\begin{array}{r}
\frac{\partial^{m}}{\partial x_{1}^{m}} G(f(x)) \sim G^{m}(f(x))\left(\frac{\partial f}{\partial x_{1}}\right)^{m}+\text { lower order terms } \sim\left(|x|^{-\alpha-1}\right)^{m} \\
\text { as }|x| \rightarrow 0,
\end{array}
$$

at least if $G^{(m)}(t) \nrightarrow 0$ as $t \rightarrow \infty$. Compare this with

$$
\frac{\partial^{m} f}{\partial x_{1}^{m}}(x) \sim|x|^{-\alpha-m} \quad \text { as }|x| \rightarrow 0 .
$$

Hence, superpositions with even very smooth functions $G$ create stronger singularities in the derivatives of order $m \geq 2$. Inequality (2.16) is proved in Sickel [24].

2.4. The counterexample of Dahlberg. As mentioned in the introduction more than ten years ago Dahlberg [7] proved: If $G \in C^{2}\left(\mathbb{R}^{1}\right)$ such that $G(f) \in W_{p}^{m}$ for 
all $f \in \widetilde{W}_{p}^{m}$, where $1+1 / p<m<n / p$, then $G$ is a linear function. The example he used is a function of the type

$$
f(x)=\sum_{j=1}^{\infty} j^{\beta} u\left(j^{\alpha}\left(x-z^{j}\right)\right),
$$

where $u \in C_{0}^{\infty}, u(x)=u\left(x_{1}, \ldots, x_{n}\right)=x_{1}$ if $|x| \leq 1, u(x)=0$ if $|x| \geq 2,\left\{z^{j}\right\}_{j=1}^{\infty}$ is an appropriate sequence in $\mathbb{R}^{n}$ and $\alpha, \beta$ are positive real numbers.

By using the same example the degeneracy result was extended to $\widetilde{B}_{p, q}^{s}$ and $\widetilde{F}_{p, q}^{s}$ by Bourdaud [4] and Runst [20]. The problem of measuring this loss of smoothness was first treated in Sickel [24]. Again we applied the construction (2.18).

Theorem 5 ([24]). Let $1<s<n / p$. Let $\tau>0$. Let $G$ be $\tau$-periodic, sufficiently smooth, and non-trivial. Then for all $\varepsilon>0$ there exists $f_{\varepsilon} \in \widetilde{H}_{p}^{s}$ (with arbitrarily small support) such that $G\left(f_{\varepsilon}\right) \notin H_{p}^{\varrho+\varepsilon}$.

Remark 7. Theorem 5 proves that Theorem 4(ii) is sharp in the sense that the exponent $\varrho$ cannot be improved in general.

We make a simple observation concerning the loss of smoothness. Let $n$ and $p$ be fixed such that $n / p>1$. We define

$$
d(s)=s-\varrho(s, n / p) .
$$

One easily checks $\lim _{s \downarrow} d(s)=\lim _{s \uparrow n / p} d(s)=0$. Furthermore, $\varrho<s$ if $1<s<$ $n / p$ and $d(s)$ is concave there. Hence, $d(s)$ has a maximum on $(1, n / p)$. It is taken at the point

$$
s_{0}=n / p-\sqrt{n / p}+1,
$$

and

$$
d\left(s_{0}\right)=(\sqrt{n / p}-1)^{2}
$$

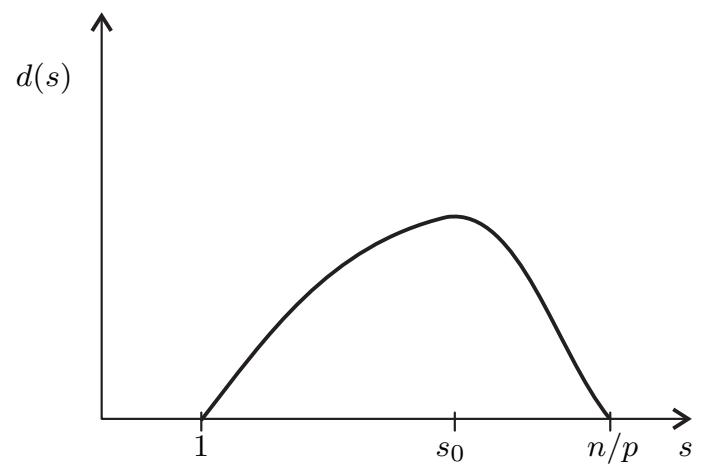

Fig. 2

(cf. Fig. 2). Consequently, $d(s)$ can become arbitrarily large if $n / p \rightarrow \infty$. To make the behaviour of $T_{G}$ more clear, we draw a further figure. 


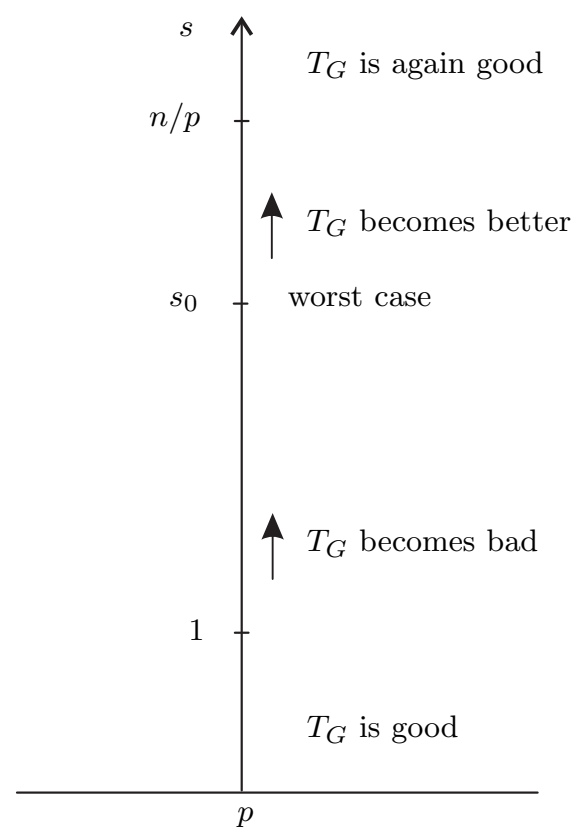

Fig. 3

Here " $T_{G}$ is good" means $T_{G}$ maps a space $H_{p}^{s}$ into itself and " $T_{G}$ becomes bad" means $d(s)$ is increasing. On the other hand, " $T_{G}$ becomes better" is used for $d(s)$ decreasing.

Figure 3 shows that the behaviour of nonlinear operators can be completely different from that of linear ones. Since $T_{G}$ is good on $H_{p}^{s}, s>n / p$, and on $H_{p}^{s}$, $s \leq 1$, by interpolation one would also expect a good behaviour with respect to $\left[H_{p}^{s_{0}}, L_{p}\right]_{\theta}=H_{p}^{s}, 0<\theta<1, s=(1-\theta) s_{0}$ (cf. Triebel [29]). But this is false by Fig. 3.

Remark 8. Note that $\tau$-periodicity of $G$ in Theorem 5 is not necessary. One needs the existence of a sequence of disjoint intervals $\left\{I_{j}\right\}_{j=1}^{\infty}$ with

$$
\begin{gathered}
\inf _{j}\left|I_{j}\right| \geq A>0, \\
I_{j} \subset\left\{t:\left|G^{(m+1)}(t)\right| \geq B>0\right\},
\end{gathered}
$$

where $m+1=[\varrho+1]$ (integer part) for some $A, B>0$.

Since a function like $\left(1+t^{2}\right)^{-\alpha}, \alpha>0$, cannot satisfy (2.21), (2.22), the following degeneracy result is also of interest.

Theorem 6 ([24]). Let $1+1 / p<s<n / p$. Let

$$
\varrho^{*}\left(s, \frac{n}{p}\right)=\varrho^{*}=\frac{\frac{n}{p}+\frac{1}{p}\left(\frac{n}{p}-s\right)}{\frac{n}{p}-s+1} .
$$


Put $m=\left[\varrho^{*}\right]$ (integer part). Let $G$ be sufficiently smooth and let $G^{(m+1)}$ be nontrivial. Then for any $\varepsilon>0$ there exists $f_{\varepsilon} \in \widetilde{H}_{p}^{s}$ (with arbitrarily small support) such that $G(f) \notin H_{\bar{p}}^{\varrho^{*}+\varepsilon}$.

R e mark 9. A short calculation gives $1+1 / p<\varrho^{*}<s, \varrho<\varrho^{*}$ if $1+1 / p<s<$ $n / p$, so for any non-trivial $G$ we have some loss of smoothness after superposition.

R e mark 10. Positive results for the number $\varrho^{*}$, i.e. improvements on Theorem 4(ii) under additional assumptions on $G$ are not known to the author.

Rem ark 11. Let $\Omega \subseteq \mathbb{R}^{n}$ be a bounded $C^{\infty}$-domain. Let

$$
\begin{gathered}
H_{p}^{s}(\Omega)=\left\{f \in L_{p}(\Omega): \exists g \in H_{p}^{s}\left(\mathbb{R}^{n}\right) \text { such that }\left.g\right|_{\Omega}=f\right\}, \\
\left\|f\left|H_{p}^{s}(\Omega)\left\|=\inf _{\left.g\right|_{\Omega}=f}\right\| g\right| H_{p}^{s}\left(\mathbb{R}^{n}\right)\right\| .
\end{gathered}
$$

Theorems 5 and 6 are also applicable in this situation, since we can make the support of $f_{\varepsilon}$ as small as we want.

2.5. Boundedness of superposition operators in Sobolev spaces of fractional order $s \leq 1+1 / p$. Theorems 5 and 6 make it plausible that under additional conditions on $G$ the operator $T_{G}$ maps $H_{p}^{s}$ into $H_{p}^{s}$ if $s \leq 1+1 / p$.

Theorem 7 ([25]). Let $1<p<2$. Let $0 \leq t<s \leq 2 / p$. Let $G$ be a function with

(i) $G(0)=0$

(ii) $G^{\prime \prime} \in L_{1}\left(\mathbb{R}^{1}\right)$.

Then there exists a constant $c$ such that

$$
\left\|G(f)\left|H_{p}^{t}\|\leq c\| f\right| H_{p}^{s}\right\| \quad \text { for all } f \in \widetilde{H}_{p}^{s} .
$$

Remark 12. Theorem 7 is a consequence of the following result of Bourdaut [4]: If $G$ is a function with properties (i) and (ii), then there exists a constant $c$ such that

$$
\left\|G(f)\left|W_{1}^{2}\|\leq c\| f\right| \widetilde{W}_{1}^{2}\right\| \quad \text { for all } f \in \widetilde{W}_{1}^{2} .
$$

In Sickel [25] a further extension of (2.27) is obtained with the help of interpolation of nonlinear operators (cf. Peetre [18], Maligranda [11]).

2.6. An overview. Our aim is to explain in three figures the different behaviour of $T_{G}$ for $G \in C^{\infty}\left(\mathbb{R}^{1}\right), G(0)=0$. For simplicity we assume $G \not \equiv 0$.

(i) The case $n=1$. In that case we have a very simple and nice behaviour shown in Fig. 4 (cf. Theorem 4). Here $A$ stands for any space $H_{p}^{s}$, where the couple $(s, 1 / p)$ is taken from the shaded region.

(ii) The case $n=2$. As a consequence of Theorems 4 and 7 we obtain the situation as in Fig. 5. 


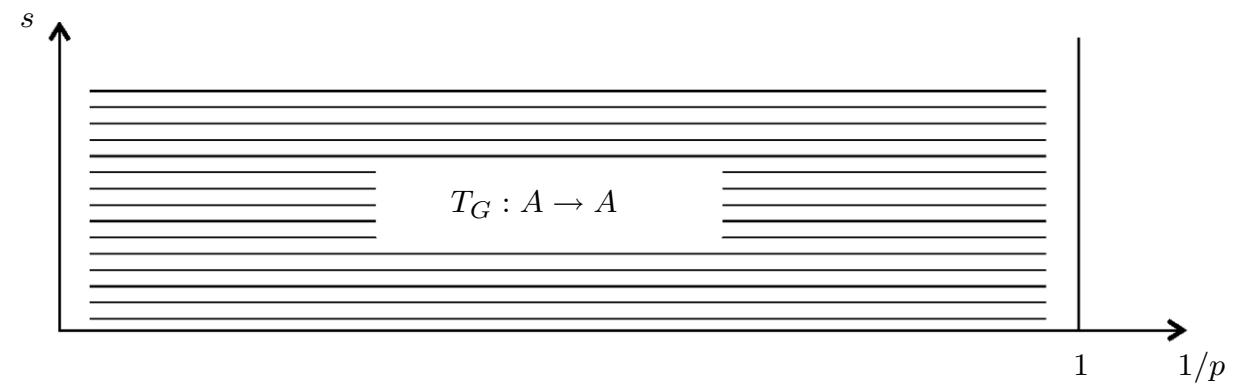

Fig. 4

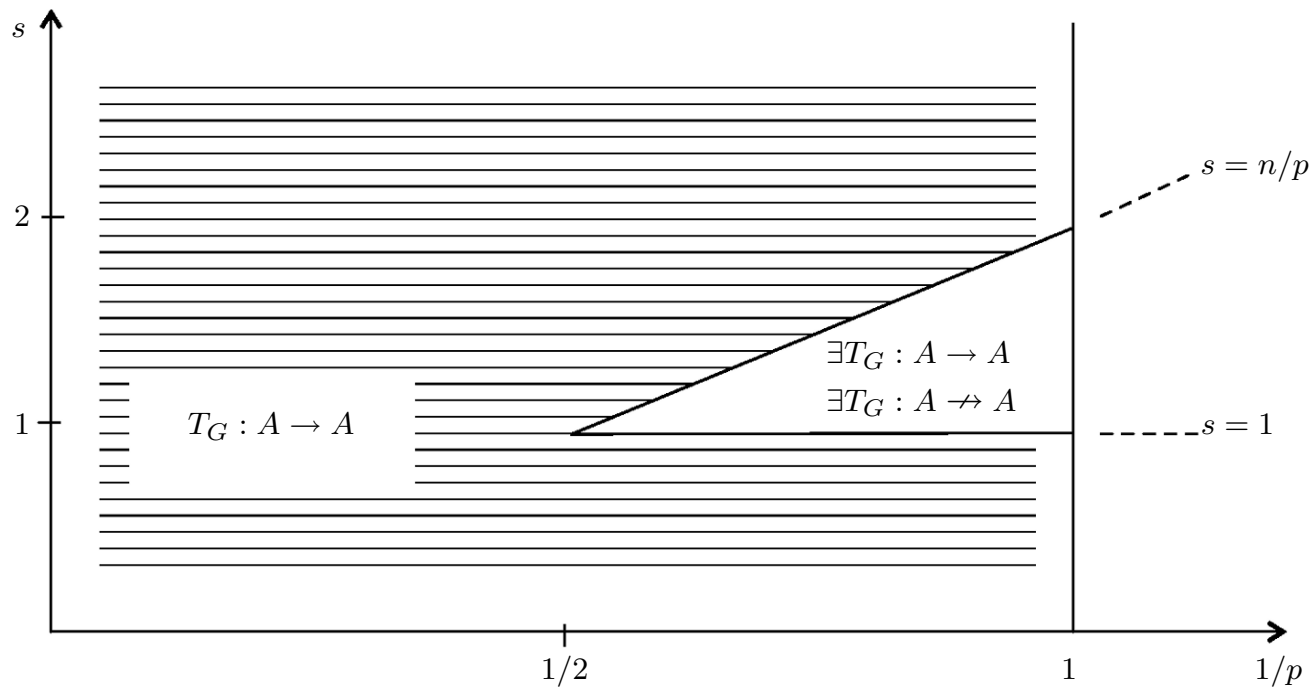

Fig. 5

In the non-shaded region $1<p<2,1<s<2 / p$ the symbol " $\exists T_{G}: A \nrightarrow A$ " is used for the fact that there exists some $G$ (cf. Theorem 4 and Remark 8) such that $T_{G}$ does not map $A$ into $A$, while " $\exists T_{G}: A \rightarrow A$ " means that there exists some $G$ (cf. Theorem 7 ) such that $T_{G}$ maps $A$ into $A$.

(iii) The general case $n \geq 3$. Now we have to use Theorems $4-7$ (see Fig. 6).

In the region $1<p<n, \max (1,2 / p)<s<1+1 / p$ it is an open problem whether there exists some $G \in C^{\infty}\left(\mathbb{R}^{1}\right)$ such that $T_{G}: A \rightarrow A$ holds. Note that for $1 /(n-1)<1 / p<1,1+1 / p<s<n / p$ we have $T_{G}: A \nrightarrow A$ for any $G \not \equiv 0$.

2.7. Some further results on boundedness of superposition operators

2.7.1. Moser-type inequalities. It is known that by restriction to bounded functions one can improve several of the results collected in 2.1-2.3. A first example is the embedding

$$
\left(W_{p}^{m} \cap L_{\infty}\right) \circ\left(W_{p}^{m} \cap L_{\infty}\right) \hookrightarrow W_{p}^{m}, \quad m=1,2, \ldots,
$$




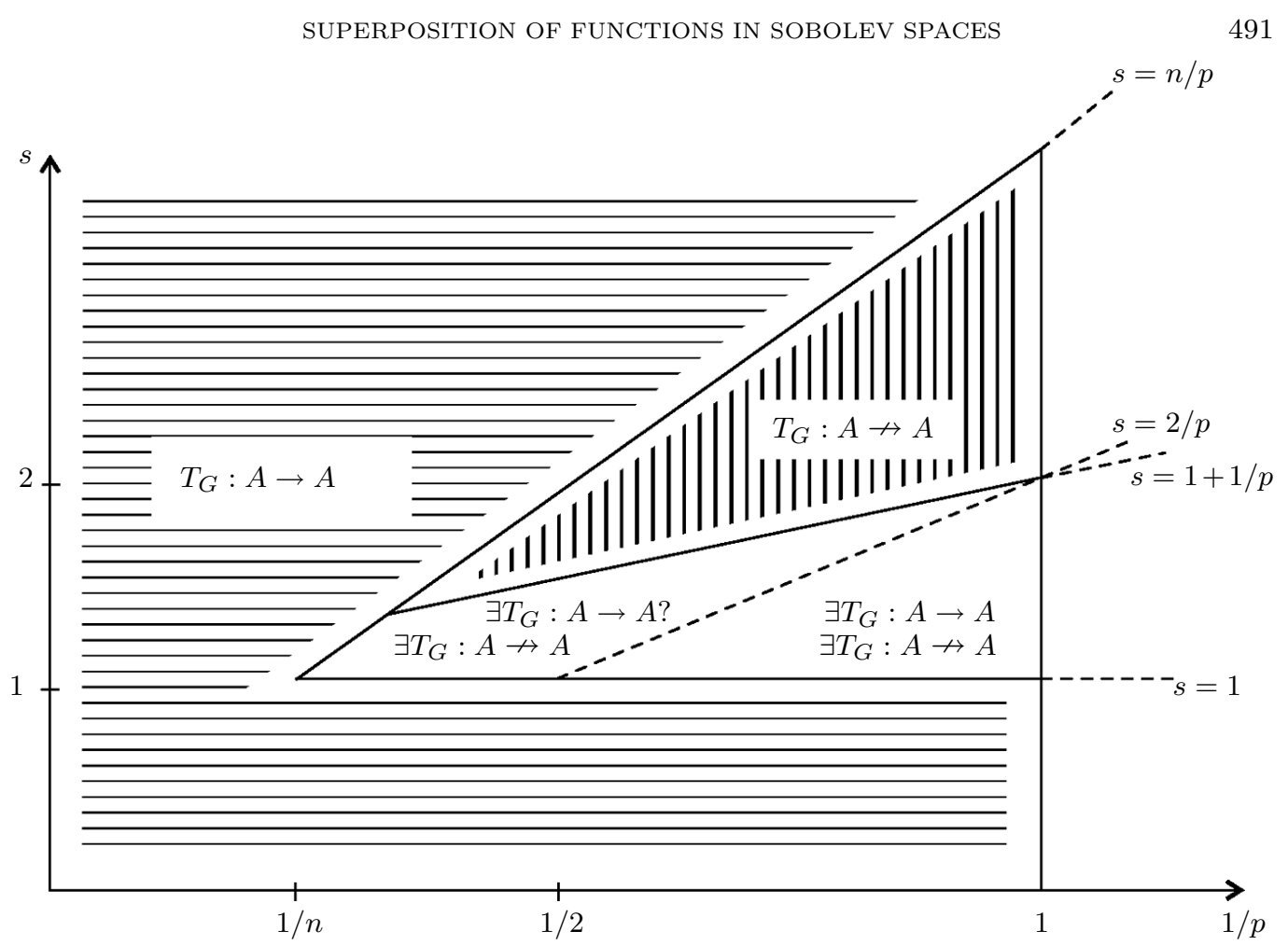

Fig. 6

which holds true without the restriction $m>n / p$ (cf. Nirenberg [17]). Later on Moser [16] dealt with some extensions. Recall that the results in 2.1-2.3 are based on assertions on pointwise multipliers. So, (2.28) gives some hope of improving Theorems 1-4.

Theorem 8 ([20], [24], [25]). Let $G \in C^{\infty}\left(\mathbb{R}^{1}\right), G(0)=0$.

(i) Let $m=2,3, \ldots$ Then there exists a constant $c$ such that

$$
\left\|f^{m}\left|H_{p}^{s}\|\leq c\| f\right| H_{p}^{s}\right\|\left\|f \mid L_{\infty}\right\|^{m-1} \quad \text { for all } f \in H_{p}^{s} \cap L_{\infty} .
$$

(ii) Let $\mu>1$ and $s<\mu$. Then there exist a constant $c$ such that

$$
\left\||f|^{\mu}\left|H_{p}^{s}\|\leq c\| f\right| H_{p}^{s}\right\|\left\|f \mid L_{\infty}\right\|^{\mu-1} \quad \text { for all } f \in H_{p}^{s} \cap L_{\infty} .
$$

(iii) There exists a constant $c$ such that

$$
\begin{aligned}
& \left\|G(f) \mid H_{p}^{s}\right\| \leq c\left(\left\|f\left|H_{p}^{s}\|+\| f\right| H_{p}^{s}\right\|\left\|f \mid L_{\infty}\right\|^{\max (0, s-1)}\right) \\
& \quad \text { for all } f \in \widetilde{H}_{p}^{s} \cap L_{\infty} .
\end{aligned}
$$

Re mark 13. Further contributions to this subject can be found in Peetre [18] and Adams-Frazier [1]. The first deals with $B_{p, q}^{s} \cap L_{\infty}$ (Besov spaces), whereas the second is concerned with the action of $T_{G}$ on $H_{p}^{s} \cap \mathrm{BMO}$. 
2.7.2. An improvement of the integrability properties. In Sickel $[24,25]$ we considered the possibility that one can improve the results of the preceding subsections concerning integrability properties. We ask now for an embedding

$$
T_{G}\left(H_{p_{0}}^{s}\right) \hookrightarrow H_{p_{1}}^{s}, \quad p_{0} \geq p_{1} .
$$

It is not our aim to treat (2.32) in its full generality. We only mention the following two interesting lemmata.

Lemma 1. Let $0<s<n / p$.

(i) Let $m=2,3, \ldots$ and let

$$
1<r<\infty, \quad \frac{p}{m} \leq r \leq \frac{n}{s+m(n / p-s)} .
$$

Then there exists a constant $c$ such that

$$
\left\|f^{m}\left|H_{r}^{s}\|\leq c\| f\right| H_{p}^{s}\right\|^{m} \quad \text { for all } f \in H_{p}^{s} .
$$

(ii) Let $\max (1, s)<\mu$ and let

$$
1<r<\infty, \quad \frac{p}{\mu} \leq r \leq \frac{n}{s+\mu(n / p-s)} .
$$

Then there exists a constant $c$ such that

$$
\left\||f|^{\mu}\left|H_{r}^{s}\|\leq c\| f\right| H_{p}^{s}\right\|^{\mu} \quad \text { for all } f \in H_{p}^{s} .
$$

Lemma 2. Let $\Omega$ be a bounded $C^{\infty}$-domain. Let $G \in C^{\infty}\left(\mathbb{R}^{1}\right), G(0)=0$. Let $0<s<n / p$ and

$$
1<r<\frac{n}{s+\max (1, s)(n / p-s)} .
$$

Then there exists a constant $c$ such that

$$
\begin{aligned}
& \left\|G(f) \mid H_{r}^{s}(\Omega)\right\| \leq c\left(\left\|f\left|H_{p}^{s}(\Omega)\|+\| f\right| H_{p}^{s}(\Omega)\right\|^{\max (1, s)}\right) \\
& \text { for all } f \in \widetilde{H}_{p}^{s}(\Omega) \text {. }
\end{aligned}
$$

Remark 14. From the embedding relations for $H_{p}^{s}$-spaces we know that

$$
H_{r}^{s} \hookrightarrow H_{p}^{s_{\mu}}, \quad r=\frac{n}{s+\mu(n / p-s)}>1,
$$

and $H_{r+\varepsilon}^{s} \hookrightarrow H_{p}^{s_{\mu}+\delta}, \varepsilon>0, \delta=\delta(\varepsilon)>0$ (cf. Triebel [30]). Thus, the number $r$ cannot be improved since $s_{\mu}$ is best possible (cf. Theorem 2, Remarks 3 and 5).

Remark 15. Of course, (2.33), (2.35), and (2.37) also imply further restrictions on $s$. For instance, from (2.33), (2.35) we find

$$
s>\frac{n}{p}-\frac{1}{\mu-1}\left(n-\frac{n}{p}\right)
$$

to guarantee $n /(s+\mu(n / p-s))>1$. Using a similar condition to (2.40), Cazenave and Weissler [6] proved a corresponding statement for homogeneous Besov spaces. 
2.7.3. Minimal smoothness conditions on $G$. It is more or less clear that $G \in C^{\infty}\left(\mathbb{R}^{1}\right)$ is far from optimal. A more detailed examination of our approach yields that $G \in C^{r}\left(\mathbb{R}^{1}\right)$ with $r>s$ (in the case of (2.14), (2.27)) or $r>\varrho$ (in the case of (2.15)) is always sufficient. Moreover, the constants which appear in these inequalities have the form $c=c^{\prime}\left\|G \mid C^{r}\left(\mathbb{R}^{1}\right)\right\|, c^{\prime}$ independent of $G$. However, this is not optimal either. For Sobolev spaces $W_{p}^{1}$, it is known (cf. Marcus and Mizel [12]) that $T_{G}$ maps $W_{p}^{1}$ into $W_{p}^{1}$ if and only if

(i) $G$ is locally Lipschitz continuous if either $p>n$, or $n=1$ and $p \geq 1$,

(ii) $G$ is uniformly Lipschitz continuous if $p<n$.

Also the result of Bourdaud [4] mentioned in Remark 12 cannot be improved, at least if $W_{1}^{2} \hookrightarrow L_{\infty}$. A more general result in this direction is the following.

TheOrem 9 ([25]). To have an embedding

$$
T_{G}\left(H_{p}^{s}\right) \hookrightarrow H_{p}^{s}
$$

it is necessary that $G \in H_{p}^{s, \text { loc }}\left(\mathbb{R}^{1}\right)$.

Remark 16. In this connection let us refer to Szigeti [28] who stated that

$$
T_{G}\left(W_{p}^{m}([a, b])\right) \hookrightarrow W_{p}^{m}([a, b]), \quad-\infty<a<b<\infty,
$$

if $G \in W_{p}^{m}\left(\mathbb{R}^{1}\right)$ and $m \geq 2$. Moreover, he investigated the example $f(x)=$ $|x|^{\alpha-1 / p} \psi(x), x \in \mathbb{R}^{1}, \alpha>1 / p$, and $G(t)=|t|^{\beta-1 / p} \psi(t), t \in \mathbb{R}^{1}, \beta>1 / p$ (cf. $(1.3),(1.5))$. The superposition results in

$$
\begin{aligned}
& G(f(x)) \sim|x|^{(\alpha-1 / p)(\beta-1 / p)} \quad \text { near zero, which gives } \\
& G(f(x)) \in H_{p}^{r}\left(\mathbb{R}^{1}\right), \quad r<(\alpha-1 / p)(\beta-1 / p)+1 / p .
\end{aligned}
$$

Because of $f \in H_{p}^{s}\left(\mathbb{R}^{1}\right), s<\alpha$, it is necessary to have

$$
(\alpha-1 / p)(\beta-1 / p)+1 / p>\alpha
$$

to guarantee the embedding (2.42). This means $\beta-1 / p>1$. Hence, in that case $G \in H_{p}^{s}\left(\mathbb{R}^{1}\right), s>1+1 / p$, is necessary to have $(2.42)$.

In the literature some attention is also paid to the mappings $f \rightarrow|f|$ or equivalently to $f \rightarrow \max (0, f)=f_{+}, f \rightarrow \min (0, f)=f_{-}$. As a supplement to Theorem 7 and to the above-mentioned result of Marcus and Mizel [12] we have proved the following in Runst-Sickel [22]:

TheOrem 10. Let $\varepsilon>0$. Let $1<p<2$. Let $0 \leq s<2 / p$. Then there exists a constant $c_{\varepsilon}$ such that

$$
\left\||f|\left|H_{p}^{s}\left\|\leq c_{\varepsilon}\right\| f\right| H_{p}^{s+\varepsilon}\right\| \quad \text { for all } f \in H_{p}^{s+\varepsilon} \text {. }
$$

Remark 17. The proof in [22] is based on the fact that the translates and dilates of the hut function $N$ (see Fig. 7) form a dense set in $H_{p}^{s}, 1<p<\infty$, $0 \leq s<1+1 / p$. Furthermore, one can use the formula $\left|\sum_{j} \alpha_{j} N(t-j)\right|=$ $\sum_{j}\left|\alpha_{j}\right| N(t-j), t \in \mathbb{R}^{1}$. 


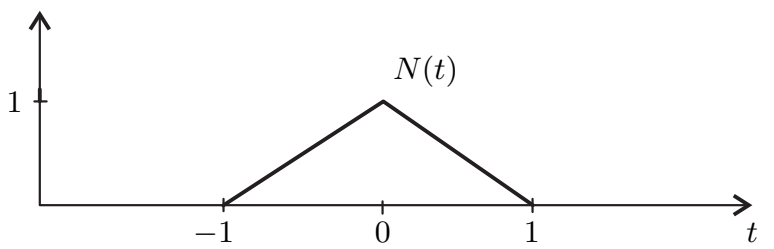

Fig. 7

2.7.4. $\mathbb{R}^{m} \rightarrow \mathbb{R}^{1}$ functions $G$. Using similar ideas to the case of $\mathbb{R}^{1} \rightarrow \mathbb{R}^{1}$ functions one obtains the following generalizations of Theorems 4 and 8 .

Theorem $11([25])$. Let $G: \mathbb{R}^{m} \rightarrow \mathbb{R}^{1}, G(0, \ldots, 0)=0$ and $G \in C^{\infty}\left(\mathbb{R}^{n}\right)$.

(i) Let $s \geq 0$. Then there exists a constant $c$ such that

$$
\begin{aligned}
&\left\|G\left(f_{1}, \ldots, f_{m}\right) \mid H_{p}^{s}\right\| \\
& \leq c \max _{i=1, \ldots, m}\left(\left\|f_{i}\left|H_{p}^{s}\|+\| f_{i}\right| H_{p}^{s}\right\|\left\|f_{i} \mid L_{\infty}\right\|^{\max (0, s-1)}\right)
\end{aligned}
$$

for all $\left(f_{1}, \ldots, f_{m}\right) \in\left(\widetilde{H}_{p}^{s} \cap L_{\infty}\right)^{m}$.

(ii) Let $1<s<n / p$. Let $\varrho$ be defined as in (2.15). Then there exists a constant c such that

$$
\left\|G\left(f_{1}, \ldots, f_{m}\right) \mid H_{p}^{\varrho}\right\| \leq c \max _{i=1, \ldots, m}\left(\left\|f_{i}\left|H_{p}^{s}\|+\| f_{i}\right| H_{p}^{s}\right\|^{\varrho}\right)
$$

for all $\left(f_{1}, \ldots, f_{m}\right) \in\left(\widetilde{H}_{p}^{s}\right)^{m}$.

(iii) Let $0 \leq s \leq 1$. Then there exists a constant $c$ such that

$$
\left\|G\left(f_{1}, \ldots, f_{m}\right) \mid H_{p}^{s}\right\| \leq c \max _{i=1, \ldots, m}\left(\left\|f_{i} \mid H_{p}^{s}\right\|\right)
$$

for all $\left(f_{1}, \ldots, f_{m}\right) \in\left(\widetilde{H}_{p}^{s}\right)^{m}$.

3. Continuity and differentiability of $T_{G}$. In most applications continuity and smoothness properties of $T_{G}$ are also of interest.

3.1. Continuity of $T_{G}$. The following simple trick yields the continuity of $T_{G}$ as a consequence of its boundedness. We apply the interpolation inequality

$$
\left\|G(f)-G(g)\left|H_{p}^{s}\|\leq\| G(f)-G(g)\right| H_{p}^{s_{0}}\right\|^{1-\theta}\left\|G(f)-G(g) \mid L_{p}\right\|^{\theta},
$$

where $0<\theta<1, s=(1-\theta) s_{0}$ (cf. Triebel $[29,30]$ ). Then the $L_{p}$ continuity of $T_{G}$ in connection with its $H_{p}^{s_{0}}$ boundedness yield the continuity of $T_{G}$ as a mapping from $H_{p}^{s_{0}}$ into $H_{p}^{s}$. By choosing $\theta$ sufficiently small, the defect $s_{0}-s$ can be made arbitrarily small.

A little more elegant is the following application of Theorem 11, which works for bounded functions. We use the identity

$$
\begin{aligned}
G(f)-G(g) & =\frac{G(f)-G(g)}{f-g}(f-g)-G^{\prime}(0)(f-g)+G^{\prime}(0)(f-g) \\
& =H(f, g)(f-g)+G^{\prime}(0)(f-g)
\end{aligned}
$$


and the fact that $H_{p}^{s} \cap L_{\infty}$ is a multiplication algebra. This leads to the following theorem (cf. Franke-Runst [10], Drabek-Runst [8], Sickel [25]).

Theorem 12. Let $G \in C^{\infty}\left(\mathbb{R}^{1}\right), G(0)=0$. Then $T_{G}$ is locally Lipschitz continuous as a mapping of $\widetilde{H}_{p}^{s} \cap L_{\infty}$ into itself. Moreover,

$$
\begin{array}{r}
\left\|G(f)-G(g) \mid H_{p}^{s}\right\| \\
\leq c\left(\| f - g | H _ { p } ^ { s } \| + \| f - g | L _ { \infty } \| \operatorname { m a x } \left(\left\|f\left|H_{p}^{s}\|+\| f\right| H_{p}^{s}\right\|\left\|f \mid L_{\infty}\right\|^{\max (0, s-1)},\right.\right. \\
\left.\left\|g\left|H_{p}^{s}\|+\| g\right| H_{p}^{s}\right\|\left\|g \mid L_{\infty}\right\|^{\max (0, s-1)}\right)
\end{array}
$$

for all $f, g \in \widetilde{H}_{p}^{s} \cap L_{\infty}$.

3.2. Differentiability. Sometimes also differentiability properties of $T_{G}$ are of interest. Here we only present the following result.

TheOREM 13 ([25]). Let $G$ be an infinitely differentiable function on $\mathbb{R}^{1}$. Let $\Omega$ be a bounded $C^{\infty}$-domain. Let $s>n / p$. Then the operator $T_{G}$ is infinitely differentiable as a mapping from $H_{p}^{s}(\Omega)$ into $H_{p}^{s}(\Omega)$. We have

$$
\left(T_{G}(f)\right)^{(j)}\left[g_{1}, \ldots, g_{j}\right]=G^{(j)}(f) g_{1} \circ \ldots \circ g_{j}, \quad j=1,2, \ldots,
$$

$f \in H_{p}^{s}(\Omega), g_{1}, \ldots, g_{j} \in H_{p}^{s}(\Omega)$. Moreover,

$$
\left\|G(f+g)-\sum_{j=0}^{N} \frac{G^{(j)}(f)}{j !} g^{j}\left|H_{p}^{s}(\Omega)\|\leq c\| g\right| H_{p}^{s}(\Omega)\right\|^{N+1}\left(1+\left\|g \mid H_{p}^{s}(\Omega)\right\|\right)
$$

for all $f, g \in H_{p}^{s}$ and all $N=1,2, \ldots$

A final remark. In Runst [19-21], Runst-Sickel [22], Triebel [31] and Sickel [23-25] boundedness and continuity of superposition operators are investigated in the scales $B_{p, q}^{s}$ and $F_{p, q}^{s}$. On the one hand, this is a natural extension of the case treated above because of $F_{p, 2}^{s}=H_{p}^{s}$; on the other hand, $F_{p, q}^{s}$ and $B_{p, q}^{s}$ are meaningful also for $p \leq 1$. Beside some technical difficulties, also the problem itself then becomes complicated. For instance, in case $n=1$ or $n=2, G \in C^{\infty}\left(\mathbb{R}^{1}\right)$ we obtain similar figures as in the general case $n \geq 3$ (cf. Figs. 4-6), since the critical triangle starts at $(s, 1 / p)=(1,1 / n)$ (cf. Fig. 6). Also our considerations in 2.7.2 make it meaningful to deal with $p \leq 1$.

\section{References}

[1] D. R. Adams and M. Frazier, BMO and smooth truncation in Sobolev spaces, Studia Math. 89 (1988), 241-260.

[2] J. Appell and P. Zabre ̌̌ko, Nonlinear Superposition Operators, Cambridge Univ. Press, Cambridge 1990.

[3] N. Aronszajn and K. T. Smith, Theory of Bessel potentials. I, Ann. Inst. Fourier (Grenoble) 11 (1961), 385-476.

[4] G. Bourdaud, Sur les opérateurs pseudo-différentiels à coefficients peu réguliers, Diss., Univ. de Paris Sud, 1983. 
[5] A. P. Calderón, Lebesgue spaces of functions and distributions, in: Partial Differential Equations, Proc. Sympos. Pure Math. 4, Amer. Math. Soc., 1961, 33-49.

[6] T. Cazenave and F. B. Weissler, The Cauchy Problem for the critical nonlinear Schrödinger equation in $H^{s}$, Nonlinear Anal. 14 (1990), 807-836.

[7] B. E. J. Dahlberg, A note on Sobolev spaces, in: Proc. Sympos. Pure Math. 35, Part I, Amer. Math. Soc., 1979, 183-185.

[8] P. Drabek and Th. Runst, On the existence of solutions of a semilinear elliptic boundary value problem with superlinear nonlinearities, Z. Anal. Anwendungen 9 (1990), 105-112.

[9] D. E. Edmunds and H. Triebel, Remarks on nonlinear elliptic equations of the type $\Delta u+u=|u|^{p}+f$ in bounded domains, J. London Math. Soc. (2) 91 (1985), 331-339.

[10] J. Franke and T. Runst, On the admissibility of function spaces of type $B_{p, q}^{s}$ and $F_{p, q}^{s}$ and boundary value problems for non-linear partial differential equations, Anal. Math. 13 (1987), 3-27.

[11] L. Maligranda, Integration of locally Hölder operators, Studia Math. 78 (1984), 289-296.

[12] M. Marcus and V. J. Mizel, Complete characterization of functions which act via superposition on Sobolev spaces, Trans. Amer. Math. Soc. 251 (1979), 187-218.

[13] J. Marschall, Pseudo-differential operators with nonregular symbols, thesis, FU Berlin, 1985.

[14] Y. Meyer, Remarques sur un théorème de J. M. Bony, Rend. Circ. Mat. Palermo (2) Suppl. 1 (1981), 1-20.

[15] S. Mizohata, Lectures on the Cauchy Problem, Tata Institute, Bombay 1965.

[16] J. Moser, A rapidly convergent iteration method and non-linear differential equations. I, Ann. Scuola Norm. Sup. Pisa 20 (2) (1966), 265-315; II, ibid. 20 (3) (1966), 499-535.

[17] L. Nirenberg, On elliptic partial differential equations, ibid. 13 (1959), 115-162.

[18] J. Peetre, Interpolation of Lipschitz operators and metric spaces, Mathematica (Cluj) 12 (35) (1970), 325-334.

[19] T. Runst, Paradifferential operators in spaces of Triebel-Lizorkin and Besov type, Z. Anal. Anwendungen 4 (1985), 557-573.

[20] - Mapping properties of non-linear operators in spaces of Triebel-Lizorkin and Besov type, Anal. Math. 12 (1986), 323-346.

[21] - Solvability of semilinear elliptic boundary value problems in function spaces, in: Surveys on Analysis, Geometry and Mathematical Physics, Teubner-Texte Math. 117, Teubner, Leipzig 1990, 198-292.

[22] T. Runst and W. Sickel, Mapping properties of $T: f \rightarrow|f|$ in Besov-Triebel-Lizorkin spaces and an application to a nonlinear boundary value problem, preprint.

[23] W. Sickel, On pointwise multipliers in Besov-Triebel-Lizorkin spaces, in: Seminar Analysis of the Karl-Weierstrass-Institute 1985/86, Teubner-Texte Math. 96, Teubner, Leipzig 1987, 45-103.

[24] - On boundedness of superposition operators in spaces of Triebel-Lizorkin type, Czechoslovak Math. J. 39 (114) (1989), 323-347.

[25] -, Abbildungseigenschaften von Nemytckii-Operatoren in Besov-Triebel-Lizorkin-Räumen und ausgewählte Anwendungen, manuscript.

[26] E. M. Stein, Singular Integrals and Differentiability Properties of Functions, Princeton Univ. Press., Princeton 1970.

[27] R. S. Strichartz, Multipliers on fractional Sobolev spaces, J. Math. Mech. 16 (1967), 1031-1060.

[28] F. Szigeti, On Niemitzki operators in Sobolev spaces, Z. Angew. Math. Mech. 63 (5) (1983), T332.

[29] H. Triebel, Interpolation Theory, Function Spaces, Differential Operators, NorthHolland, Amsterdam, and Deutscher Verlag Wiss., Berlin 1978. 
[30] H. Triebel, Theory of Function Spaces, Akad. Verlagsges. Geest \& Portig K. G., Leipzig 1983 and Birkhäuser, Basel 1983.

[31] —, Mapping properties of non-linear operators generated by holomorphic $\phi(u)$ in function spaces of Besov-Sobolev-Hardy type. Boundary value problems for elliptic differential equations of type $\Delta u=f(x)+\Phi(u)$, Math. Nachr. 117 (1984), 193-213.

[32] M. Yamazaki, A quasi-homogeneous version of paradifferential operators, I. Boundedness on spaces of Besov type, J. Fac. Sci. Univ. Tokyo Sect. IA Math. 33 (1986), 131-174. 